\title{
BMJ Open Exploring healthcare providers' perceptions of mental health amid COVID-19 pandemic in obstetrics and gynaecology department of a tertiary care public sector hospital of Karachi, Pakistan: an exploratory qualitative study protocol
}

\author{
Haleema Yasmin (D) , ${ }^{1}$ Afreen Sadia, ${ }^{2}$ Areesha Qamar, ${ }^{1}$ Anam Shahil Feroz (D) ${ }^{2,3}$
}

\begin{abstract}
To cite: Yasmin H, Sadia A, Qamar A, et al. Exploring healthcare providers' perceptions of mental health amid COVID-19 pandemic in obstetrics and gynaecology department of a tertiary care public sector hospital of Karachi, Pakistan: an exploratory qualitative study protocol. BMJ Open 2021;11:e054010. doi:10.1136/ bmjopen-2021-054010
\end{abstract}

\section{- Prepublication history and} additional supplemental material for this paper are available online. To view these files, please visit the journal online (http://dx.doi.org/10.1136/ bmjopen-2021-054010).

HY and ASF are joint first authors.

Received 07 June 2021 Accepted 03 November 2021

Check for updates

(c) Author(s) (or their employer(s)) 2021. Re-use permitted under CC BY-NC. No commercial re-use. See rights and permissions. Published by BMJ.

For numbered affiliations see end of article.

Correspondence to

Dr Anam Shahil Feroz;

anam.shahil@mail.utoronto.ca

\begin{abstract}
Introduction In the wake of the unprecedented public health challenge of the COVID-19 pandemic, it is highly significant to recognise the mental health impact of this mounting threat on healthcare providers (HCPs) working in the obstetrics and gynaecology department. Experience from epidemics and emerging literature around COVID-19 show that the unparalleled amount of stress that HCPs are dealing with is linked with the increased burden of mental health conditions. We aim to conduct an exploratory qualitative descriptive study to assess HCPs' perceptions of mental health amid the COVID-19 pandemic in the obstetrics and gynaecology department of a public sector tertiary care hospital of Karachi, Pakistan.

Methods and analysis This study will use a qualitative descriptive approach where approximately 20-25 HCPs from the obstetrics and gynaecology department will be recruited using a purposive sampling approach. Data will be collected through semistructured interviews and it will be analysed thematically using NVivo V.12 Plus software. Ethics and dissemination Ethical approval for this study has been obtained from the Institutional Review Board Committee of Jinnah Postgraduate Medical Center hospital. The study results will be disseminated to the scientific community and the HCPs participating in the study. The findings will help us to explore the doctor's perceptions of mental health during the current pandemic of COVID-19 and its impact on their daily lives and mental well-being.
\end{abstract}

\section{INTRODUCTION}

According to WHO, there have been 250 715502 confirmed cases of COVID-19, including 5062106 deaths, globally as of 10 November 2021. ${ }^{1}$ The pandemic has not only overwhelmed the healthcare systems of low/middle-income countries such as Pakistan but also largely affected the healthcare providers (HCPs) as they work
Strengths and limitations of this study

- A robust qualitative methodology has been adopted and a diverse sample of healthcare providers (HCPs) belonging to different ethnocultural backgrounds has been included.

- An exploratory approach will provide a valuable and in-depth understanding of COVID-19 impact on the daily lives and well-being of obstetrics and gynaecology (Ob-Gyn) HCPs.

- In this study, we will include the HCPs of Ob-Gyn working in public sector hospitals therefore the sample may not be considered representative of all the HCPs working in different subspecialties.

- One limitation is that to minimise the risk of COVID-19 infection, all HCPs will be interviewed online over Zoom and hence the authors will not have the opportunity to build rapport with the respondents or obtain non-verbal cues during interviews.

in extreme conditions to provide routine healthcare services as well care specific to COVID-19. Much of the scientific literature around COVID-19 is related to disease transmission and its pathogenicity with a paucity of evidence on the mental health needs of the frontline health workforce working in tertiary care hospitals during COVID-19 pandemic. $^{2}$ Qualitative studies on the impact of COVID-19 on the mental health of HCPs from developing countries showed that the HCPs experienced a variety of psychological distress during the care of patients with COVID-19. ${ }^{3}$ Among the frontline workers, some of the specialties are very high demanding and stressful due to long working hours, high patient influx 
and constant dealing with emergencies. ${ }^{4}$ Obstetrics and gynaecology (Ob-Gyn) are one of the specialties with an increased level of stress due to constantly dealing with high-risk complex cases. ${ }^{5}$ A study conducted by Mushtaq et al in public sector tertiary care hospitals in Pakistan reported that $33 \%$ of the doctors working in the Ob-Gyn department experience severe stress due to the demanding nature of the job. ${ }^{4}$

With the advent of COVID-19 in Pakistan, all health professionals have been experiencing stress due to fear of acquiring infection, inadequate equipment for safety from contagion, isolation, exhaustion and lack of contact with family. ${ }^{6}$ A study was done by Sandesh $e t$ al in different hospitals of Karachi also showed a high prevalence of anxiety (85\%), stress (90\%) and depression (82\%) among HCPs while working in isolation wards. ${ }^{7}$ Amid COVID-19 HCPs often had to manage pregnant women with COVID-19 in the absence of adequate resources and an unclear understanding of diseases pattern, prognosis and treatment among pregnant women also adds to the uncertainty of the HCPs. ${ }^{8}$ Lack of clarity of the virus impact on maternal and fetal outcomes, chances of vertical transmission of the infections, lack of HCPs during COVID-19, shifts pattern and rapidly changing management protocols and guidelines also adds up to the stress. ${ }^{26}$ All these factors are causing further mental health problems which not only affect HCPs working potential but also disturb their overall well-being. ${ }^{9}$

The constant pressure healthcare workers are experiencing could generate psychological issues of anxiety, fear, panic attacks, stigma and avoidance of contact, sleep disturbances, helplessness, interpersonal social isolation from family social support and concern regarding contagion exposure to their friends and family. The health professionals hesitate to seek counselling and psychotherapeutic interventions due to fear of labelling, stigmatisation and discrimination potentially. ${ }^{10-13}$ The mental health of HCP of Ob-Gyn has been one of the important issues during the recent pandemic of COVID-19 as psychological distress may prone these HCPs to a higher risk of medical errors affecting the quality of services given to pregnant women. ${ }^{6}$ Exploring the mental health issues of obstetricians and gynaecologist is thus important for maximising the working capability and efficiency towards patient's care and to devising effective strategies to support the mental health of these HCPs.

Considering the existing situation, we aim to explore HCPs' perceptions of mental health amid the COVID-19 pandemic in the Ob-Gyn department of the public sector hospital of Karachi, Pakistan. The study will be essential in understanding the impact of this pandemic on mental health, well-being and working potential of obstetricians and gynaecologists, and devising coping strategies and intervention for these HCPs, such a study can later be extended to other hospitals of Karachi and the country subsequently.

\section{METHODS AND ANALYSIS}

\section{Study design}

This formative research will employ an exploratory qualitative descriptive research design using semistructured interviews and a purposive sampling approach. Given the aim is to understand the impact of this pandemic on the mental health, and well-being of obstetricians and gynaecologists, this design is best suited to understand the perceptions and views of HCPs working in the Ob-Gyn department of public sector tertiary care hospital. The data collection methods for this formative research will involve in-depth interviews (IDIs) with HCPs of the Ob-Gyn department.

\section{Study setting}

The study will be conducted at the Jinnah Postgraduate Medical Center (JPMC) Ob-Gyn department, which is a tertiary care public hospital in Karachi, Pakistan. JPMC serves over one million patients of low socioeconomic status per year from Karachi, Interior Sindh, Baluchistan and other areas. JPMC is a 1650 -bed tertiary care public sector hospital with over 31 departments. The annual admissions at the JPMC Ob-Gyn department exceed 26 000, and approximately 19000 deliveries take place per year, all free of charge. IDIs will be conducted with frontline doctors, nurses and paramedical staff at the Ob-Gyn department of JPMC.

\section{Data collection methods and study participants}

In-depth interviews

IDIs will be conducted with HCPs including doctors, nurses and paramedical staff who are working at the Ob-Gyn department of JPMC. Doctors, nurses and paramedical staff including nurse assistants, security guards, receptionists and operation theatre technicians will be invited to participate in their off-duty hours (table 1 ). Predominately, there are mostly female HCPs working in the Ob-Gyn department therefore our sample will mainly comprise female doctors and nurses. The study coordinator will then explain the study objectives and procedures to eligible HCPs and will obtain consent for participation in the study. Trained researchers, who are experienced in conducting qualitative research, will conduct the interviews via Zoom. Participants will be assured that their anonymity will be maintained. The interview will involve a general discussion about the COVID-19 outbreak, perceptions and experiences of caring for routine patients amid the COVID-19 pandemic, and the effect of this pandemic

\begin{tabular}{ll}
\hline Table 1 Study participants for IDls \\
\hline $\begin{array}{ll}\text { In-depth interview } \\
\text { participants }\end{array}$ & Sample range \\
\hline Doctors & $6-8$ females \\
Nurses & $6-8$ females \\
Paramedical staff & $6-8$ (4 male and 4 female) \\
\hline
\end{tabular}

IDIs, in-depth interviews. 
on health professionals' mental health, well-being and working potential. We anticipate that 20-25 IDIs will be conducted with a group of HCPs and paramedics. However, additional IDIs will be conducted until data saturation.

Data saturation is the point when no new themes emerge from the additional interviews. Data collection and analysis will be done simultaneously to determine the point of data saturation. The audio recordings will be transcribed by a transcriptionist within 24 hours of the interviews. Each IDI will approximately take between 30 and $45 \mathrm{~min}$.

\section{Sampling and eligibility criteria}

A purposive sampling technique will be used to identify and recruit IDI participants for the study. All HCPs including doctors, nurses and paramedical staff who are involved in caring for routine patients at the Ob-Gyn department of JPMC will be included in this study. Those who refuse to consent will be excluded from the study.

\section{Data collection procedure}

Separate semistructured interview guides have been developed for conducting IDIs of all the eligible participants. The interview guides will be pilot tested with a non-study sample (one clinician and one nursing staff) who have the same characteristics as the study sample but belong to a different institution. The pilot testing will offer evidence-based guidance to improve the data collection guide. Interviews are anticipated to begin in December 2020. The interviews will be conducted in the local language. The initial questions on the guide will help to explore participants' perceptions and understanding of the COVID-19 outbreak. Additional questions on this guide will assess the impact of these perceptions and their experience of caring for routine patients amid the COVID-19 pandemic. The latter part of the guide assesses the effect of this pandemic on health professionals' mental health, well-being and working potential. Interviews will be scheduled at the participant's convenient day and time. An interview guide for IDIs is shown in online supplemental annex 1.

\section{Patient and public involvement}

No patients were involved.

\section{Data analysis}

All the study data will be analysed using 'NVivo' V.12 Plus software (QSR International) to enable easy and organised retrieval of data. Our study will use conventional content analysis technique to analyse all interview transcripts. ${ }^{14}$ To ensure partcipants' confidentiality, transcripts will be anonymised before uploading it into NVivo software. To conduct conventional content analysis, the two researchers will independently read the transcripts to understand the meanings of the data and to develop an interpretation of the HCPs' perception around mental health amid the COVID-19 pandemic. During analysis, the transcribed text will be divided into 'meaning units' which will be later shortened and labelled with a 'code' without losing context of the study. Codes will be then analysed and grouped into similar categories and themes. In the final step, similar categories will be assembled under subthemes and main themes. Throughout the analysis, two independent investigators will perform the coding, and category creation and a third researchers will be involved to address discrepancies identified in the coding and category creation. Since the study focuses on exploring the mental health of HCPs amid COVID-19, the researchers will also look for in vivo codes to capture vernaculars and actual spoken words of partcipants from the transcripts.

\section{Trustworthiness of the study}

Our study will use Tracy et $a l^{15}$ and Lincoln and Guba's criteria. ${ }^{16}$ to ensure trustworthiness and methodological rigour in this qualitative inquiry. To ensure credibility in our research, our study will triangulate datain two ways: data sources triangulation and investigator triangulationto compare alternative perspective and reveal any inconsistencies. ${ }^{17}$ Our study will also perform member checking of transcripts confirm whether study findings have resonance with the HCPs' experiences. For ensuring dependability in our qualitative research, we will ensure a clear audit trail to examine the study's process and product.

\section{Ethical considerations and dissemination}

Study participants will be asked to provide informed, written consent before participation in the study. The informed consent form can be submitted by the participant via WhatsApp or email. Ethical approval for this study has been obtained from the institutional review board Committee of JPMC hospital (2020-GENL-47723). The study results will be disseminated to the scientific community and the HCPs participating in the study. The findings will help us to explore the doctor's perceptions of mental health during the current pandemic of COVID-19 and its impact on their daily lives and mental well-being.

\section{DISCUSSION}

This is the first study to explore health professionals' perceptions of mental health amid the COVID-19 pandemic in the Ob-Gyn department of the tertiary care hospital of Karachi. The research will help explore challenges faced by HCPs while caring for routine patients and COVID-19 cases alongside strategies to cope with these challenges. Broadly, the study will also attempt to understand the impact of this pandemic on the mental health, well-being and working potential of health professionals providing care to pregnant women. Also, the current pandemic provides us the opportunity to break stereotypes and stigmatisation related to mental health among HCPs and this will also enable them to seek timely advice when required. However, conducting interviews 
via Zoom due to the current pandemic situation may limit the validity of the study.

Author affiliations

${ }^{1}$ Obstetrics and Gynaecology, Jinnah Postgraduate Medical Center, Karachi,

Pakistan

${ }^{2}$ Community Health Sciences, Aga Khan University, Karachi, Pakistan

${ }^{3}$ Dalla Lana School of Public Health, Institute of Health Policy, Management and

Evaluation, University of Toronto, Toronto, Ontario, Canada

Contributors HY and ASF conceived the study. ASF, HY, AS, AQ contributed to the development of the study design and final protocols for sample selection and interviews. ASF and AS contributed to writing the manuscript. All authors reviewed and approved the final version of the paper.

Funding This study is funded by the Society of Obstetricians \& Gynaecologists of Pakistan (002.SOGP.RG.2020)

Disclaimer The funders had no role in study design, data collection and analysis, decision to publish or preparation of the manuscript.

Competing interests None declared.

Patient consent for publication Not applicable.

Provenance and peer review Not commissioned; externally peer reviewed.

Supplemental material This content has been supplied by the author(s). It has not been vetted by BMJ Publishing Group Limited (BMJ) and may not have been peer-reviewed. Any opinions or recommendations discussed are solely those of the author(s) and are not endorsed by BMJ. BMJ disclaims all liability and responsibility arising from any reliance placed on the content. Where the content includes any translated material, BMJ does not warrant the accuracy and reliability of the translations (including but not limited to local regulations, clinical guidelines, terminology, drug names and drug dosages), and is not responsible for any error and/or omissions arising from translation and adaptation or otherwise.

Open access This is an open access article distributed in accordance with the Creative Commons Attribution Non Commercial (CC BY-NC 4.0) license, which permits others to distribute, remix, adapt, build upon this work non-commercially, and license their derivative works on different terms, provided the original work is properly cited, appropriate credit is given, any changes made indicated, and the use is non-commercial. See: http://creativecommons.org/licenses/by-nc/4.0/.

\section{ORCID iDs}

Haleema Yasmin http://orcid.org/0000-0001-7343-7324

Anam Shahil Feroz http://orcid.org/0000-0003-0180-0213

\section{REFERENCES}

1 WHO. WHO coronavirus (COVID-19) Dashboard, 2021. Available: https://covid19.who.int/

2 Shah N, Raheem A, Sideris M, et al. Mental health amongst obstetrics and gynaecology doctors during the COVID-19 pandemic: results of a UK-wide study. Eur J Obstet Gynecol Reprod Biol 2020;253:90-4.

3 Galehdar N, Kamran A, Toulabi T, et al. Exploring nurses' experiences of psychological distress during care of patients with COVID-19: a qualitative study. BMC Psychiatry 2020;20:489.

4 Mushtaq M. What are the coping strategies gynaecologists employ to meet with stressful situations? J Pak Med Assoc 2015;65:857-61.

5 Wise J. Life as a physician in obstetrics and gynaecology. BMJ 2020;368:m37.

6 Vafaei H, Roozmeh S, Hessami K, et al. Obstetrics healthcare providers' mental health and quality of life during COVID-19 pandemic: multicenter study from eight cities in Iran. Psychol Res Behav Manag 2020;13:563-71.

7 Sandesh R, Shahid W, Dev K, et al. Impact of COVID-19 on the mental health of healthcare professionals in Pakistan. Cureus 2020;12:e8974.

8 Koffman J, Gross J, Etkind SN, et al. Uncertainty and COVID-19: how are we to respond? J R Soc Med 2020;113:211-6.

9 Greenberg N, Docherty M, Gnanapragasam S, et al. Managing mental health challenges faced by healthcare workers during covid-19 pandemic. BMJ 2020;368:m1211.

10 Rana W, Mukhtar S, Mukhtar S. Mental health of medical workers in Pakistan during the pandemic COVID-19 outbreak. Asian J Psychiatr 2020;51:102080.

11 Ornell F, Halpern SC, Kessler FHP, et al. The impact of the COVID-19 pandemic on the mental health of healthcare professionals. Cadernos de Saúde Pública 2020;36:e00063520.

12 Knaak S, Mantler E, Szeto A. Mental illness-related stigma in healthcare: barriers to access and care and evidence-based solutions. Healthc Manage Forum 2017;30:111-6.

13 Spoorthy MS, Pratapa SK, Mahant S. Mental health problems faced by healthcare workers due to the COVID-19 pandemic-A review. Asian J Psychiatr 2020;51:102119.

14 Vaismoradi $\mathrm{M}$, Turunen $\mathrm{H}$, Bondas $\mathrm{T}$. Content analysis and thematic analysis: implications for conducting a qualitative descriptive study. Nurs Health Sci 2013;15:398-405.

15 Tracy SJ. Qualitative quality: eight "big-tent" criteria for excellent qualitative research. Qualitative Inquiry 2010;16:837-51.

16 Lincoln YS, Guba EG. Naturalistic inquiry. 75. CA: Sage Thousand Oaks, 1985.

17 Patton MQ. Enhancing the quality and credibility of qualitative analysis. Health Serv Res 1999;34:1189. 